\title{
European Centre for Dispersion Technologies Opens
}

After a construction phase of just nine months, the European Centre for Dispersion Technologies (EZD) has been officially opened by Bavaria's State Minister of Economic Affairs. It is seen as a unique project throughout Europe and has been established by SKZ in close cooperation with industry and with considerable support by the Free State of Bavaria.

mproving dispersion technologies

in a targeted manner - this vision is now becoming a reality with the opening of the EZD in the Upper Franconia region of Bavaria. In her welcome address, the minister, Ilse Aigner, spoke of a "flagship" that would act as a role

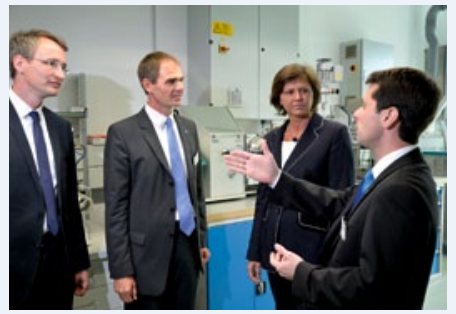

Bavarian State Minister of Economic Affairs Ilse Aigner, EZD Business Unit Manager Dr. Felipe Wolff-Fabris (right), the Mayor of Selb Ulrich Pötzsch (far left) and SKZ Institute Director Prof. Martin Bastian (2nd from left) on their tour of the new building. model far beyond the borders of Upper Franconia.

The building, with a floor space of approximately $650 \mathrm{~m}^{2}$, offers all the key development activities in the production and characterisation of dispersions as well as providing corresponding services for industry.

The variety of issues relating to the optimum dispersion of materials from a wide range of different areas is extremely high and will be one of the challenges facing Dr.-Ing. Felipe Wolff-Fabris, the manager of the new centre. "This is the first time that this increasingly important sector has had such a centre as a meeting place with close contacts to industry. It acts as the first port of call in particular for small and medium-sized companies without development capacity of their own, in which new ideas can be tested, employees can be trained and company processes can be optimised," Wolff-Fabris explained.
The Bavarian Ministry of Economic Affairs is supporting the EZD with funding amounting to $€ 5.2$ million.

\section{Interdisciplinary Institution}

The EZD is operated by SKZ in consultation with the Bavarian Ministry of Economic Affairs and local industry. "For SKZ, it is a consistent expansion of its activities in the fields of R\&D, analysis, the further training of expert staff and also the certification of companies," said Prof. Dr. Bastian, Chief Executive Officer of SKZ. He said that, with the EZD, an interdisciplinary institution had been created that offered the possibility to provide support for small and medium-sized companies and therefore to sustainably secure their competitiveness. The EZD currently employs six full-time members of staff. By 2017, it is expected to employ a total of twelve researchers as well as several interns and students, who can, for example, write to their exam thesis at the EZD.

\section{Altana Invests in Further Growth in China}

The Altana Group is signifience in China. In April, the Actega division opened a new laboratory building for coatings and sealants at its production site in Foshan. Elantas commissioned a new plant for wire enamels in Tongling, while BYK signed a contract for the purchase of a site for developing and manufacturing chemical additives in Shanghai. With these projects, the Group is investing a total of more than $€ 30$ million in the further growth of its China business. "With our investment initiative, we are aiming to further

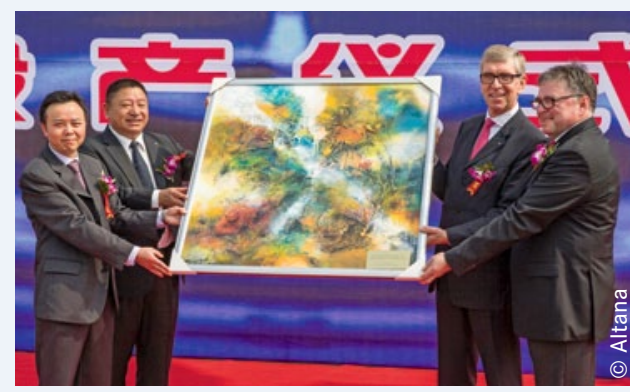

Altana CEO Dr. Matthias L. Wolfgruber (2nd from right) and Elantas Division President Dr. Guido Forstbach (right) present a painting to Elantas Tongling Managing Director Ye Lixin (left) and President Elantas Greater China Liu Linbao (2nd from left) to mark the opening of the new Elantas plant in Tongling. increase our presence in Asia with emphasis on China and take advantage of the enormous market potential here," said Dr. Matthias L. Wolfgruber, CEO of Altana AG. In 2013, Altana achieved its highest growth rate in this region in China. The Asian region as a whole grew by seven percent, driven primarily by the increased demand from China. Currently, Altana operates eight subsidiaries, five production facilities and ten laboratories in China. With 450 employees here, the company generated sales of around $€ 290$ million, or 16 percent of its entire global business, in 2013. 